\title{
FIXED POINT RESULTS FOR MAPPINGS SATISFYING CONTRACTIVE CONDITIONS BUILD ON COUPLED- $\alpha$-ADMISSIBILITY
}

\author{
Wasfi Shatanawi ${ }^{1,2}$, Kamaleldin Abodyeh ${ }^{1 \S}$ \\ ${ }^{1}$ Department of Mathematics \\ Prince Sultan University \\ Riyadh, SAUDI ARABIA \\ ${ }^{2}$ Department of Medical Research \\ China Medical University Hospital \\ China Medical University, Taichung, TAIWAN
}

\begin{abstract}
We consider in this article the notions of coupled $-\alpha$-contraction of types (I) and (II) for a mapping $F$ defined on a set $\Psi \times \Psi$. Many fixed point (FP) results in the domain of metric space are obtained using our new notions. The obtained results are used to modify some known FP theorems. Some example are given to show the authenticity of our new definitions.
\end{abstract}

AMS Subject Classification: 47H10, 54H25

Key Words: complete metric space, fixed point, $\alpha$-admissible

\section{Introduction and Preliminaries}

FP (Fixed Point) theory is one of the main subject that can be used in science to prove the existence and uniqueness of some problems. The beauty of this subject depends on constructing a sequence in a set $\Psi$ by starting with an initial point. The wideness use of this subject in many area attracted many researchers to formulate and prove many existing results in the domain of FP area.

Received: June 28, 2019

(C) 2020 Academic Publications

${ }^{\S}$ Correspondence author 
The Banach FP theorem [9] is the first remarkable result in the subject of FP theory. It is worth mentioning that many authors extended the Banach FP theorem to many directions, for example see [2, 3, 4, 7, 8, 10, 19], [22]-[27], and $[29,30,31]$.

Samet et al. [18] introduced the notion of $\alpha$-admissible for a map. It is considered as one of the attractive notion that be used to extend the Banach FP theorem to new direction. Abdeljawad [1] extended the concept of $\alpha$-admissibility to a pair of mappings to modify some theorems of Meir-Keeler type. For some work in theses notions (see [5, 6], [11]-[17], and [20, 21, 28]). In meanwhile, Karapiner et al. [14] built the notion of triangular $\alpha$-admissibility for a single mapping and studied a FP theorem of Meir-Keeler type. Salimi et al. [17] inserted a new function $\eta$ to $\alpha$-admissibilty to build the concept of $\alpha$-admissible for single mapping with respect to the function $\eta$. Recently, Hussain et al. [13] introduced a new notion of $\alpha$-admissibility and extended the Banach fixed point theorem to new version.

Now we collect all known notions of $\alpha$-admissibility.

Definition 1. ([18]) Let $\alpha$ be a nonnegative function on $\Psi \times \Psi$. A selfmapping $S$ on $\Psi$ is called $\alpha$-admissible if for any $\omega, \nu \in \Psi$ such that $\alpha(\omega, \nu) \geq 1$, then

$$
\alpha(S \omega, S \nu) \geq 1 .
$$

Karapiner [14] introduced the definition of triangular $\alpha$-admissibility for a single mapping $\alpha$.

Definition 2. ([14]) Let $\alpha: \Psi \times \Psi \rightarrow[0, \infty)$ be a function. A self-mapping $T$ on $\Psi$ is called a triangular $\alpha$-admissible mapping if

1. $T$ is $\alpha$-admissible;

2. $\alpha(\omega, v) \geq 1$ and $\alpha(v, \nu) \geq 1$ imply $\alpha(\omega, \nu) \geq 1$.

Definition 3. ([1]) Let $S, T$ be two self-mappings on $\Psi$ and $\alpha: \Psi \times \Psi \rightarrow$ $[0, \infty)$ be a function. Then the pair $(S, T)$ is called $\alpha$-admissible if for any $\omega, \nu \in \Psi$ such that $\alpha(\omega, \nu) \geq 1$, we have $\alpha(S \omega, T \nu) \geq 1$ and $\alpha(T \omega, S \nu) \geq 1$.

Definition 4. ([11]) Let $S, T: \Psi \rightarrow \Psi$ be two mappings and $\alpha, \eta: \Psi \times \Psi \rightarrow$ $\mathbf{R}$ be two functions. Then the pair $(S, T)$ is said to be $\alpha$-admissible with respect to $\eta$ if $\lambda, \nu \in \Psi$ such that $\alpha(\lambda, \nu) \geq \beta(\lambda, \nu) \Rightarrow \alpha(S \lambda, T \nu) \geq \beta(S \lambda, T \nu)$ and $\alpha(T \lambda, S \nu) \geq \beta(T \lambda, S \nu)$ 
Definition 5. ([12]) Suppose that $(\Psi, \tau)$ is a metric space and $\alpha, \eta$ are non-negative functions on $\Psi \times \Psi$. Then $\Psi$ is called an $\alpha, \eta$-complete metric space if the following condition hold: Every Cauchy sequence $\left(\lambda_{n}\right)$ in $\Psi$ such that $\alpha\left(\lambda_{n}, \lambda_{n+1}\right) \geq \eta\left(\lambda_{n}, \lambda_{n+1}\right)$, for all $n \in \mathbf{N}$, then it converges to an element in $\Psi$.

Definition 6. ([12]) Let $(\Psi, \tau)$ be a metric space and $\alpha, \eta$ be non-negative functions on $\Psi \times \Psi$. A self mapping $T$ on $\Psi$ is called an $\alpha, \eta$-continuous mapping if for any convergent sequence $\left(\lambda_{n}\right)$ in $\Psi$ that converges to an element $\lambda \in \Psi$ such that $\alpha\left(\lambda_{n}, \lambda_{n+1}\right) \geq \eta\left(\lambda_{n}, \lambda_{n+1}\right)$ for all $n \in \mathbf{N}$, then $T \lambda_{n} \rightarrow T \lambda$ as $n \rightarrow \infty$.

In this paper, we utilized the notion of $\alpha$-admissibilty to derive the notion of coupled- $\alpha$-contraction of types (I) and (II) for a mapping $F$ defined on a set $\Psi \times \Psi$. We shall also employ our new notions to produce and prove many FP results in the domain of metric space.

\section{Main Result}

We start with the following new definitions.

Definition 7. Suppose that $(\Psi, \tau)$ is a metric space and $\alpha$ is a nonnegative function on $\Psi^{4}$. Then $\Psi$ is called a coupled- $\alpha$-complete metric space if every Cauchy sequence $\left(\lambda_{n}\right)$ in $\Psi$ such that $\alpha\left(\lambda_{n}, \lambda_{n}, \lambda_{n+1}, \lambda_{n+1}\right) \geq 1$ for all $n \in \mathbf{N}$, then it converges to an element in $\Psi$.

Definition 8. Suppose that $(\Psi, \tau)$ is a metric space and $\alpha$ is a nonnegative function on $\Psi^{4}$. A mapping $F: \Psi \times \Psi \rightarrow \Psi$ is called a coupled- $\alpha$-continuous mapping if each sequence $\left(\lambda_{n}, \nu_{n}\right)$ in $\Psi \times \Psi$ with $\left(\lambda_{n}, \nu_{n}\right) \rightarrow(\lambda, \nu)$ as $n \rightarrow+\infty$ and $\alpha\left(\lambda_{n}, \lambda_{n}, \lambda_{n+1}, \lambda_{n+1}\right) \geq 1, \alpha\left(\nu_{n}, \nu_{n}, \nu_{n+1}, \nu_{n+1}\right) \geq 1$ for all $n \in \mathbf{N}$ imply that $F\left(\lambda_{n}, \nu_{n}\right) \rightarrow F(\lambda, \nu)$ as $n \rightarrow \infty$.

Example 9. Let $\Psi=\left\{\frac{1}{n}: n \in \mathbf{N}\right\}$. Define a metric $\tau$ on $\Psi \times \Psi$ via $\tau(\lambda, \nu)=|\lambda-\nu|$. Also, define the function $\alpha: \Psi^{4} \rightarrow[0,+\infty)$ via

$$
\alpha(\lambda, \nu, v, \zeta)=\left\{\begin{array}{lc}
\lambda+\nu+v+\zeta, & \text { if } \lambda, \nu, v, \zeta \in\left\{1, \frac{1}{2}\right\} \\
0, & \text { otherwise. }
\end{array}\right.
$$


Then $\Psi$ is a coupled- $\alpha$-complete which is not complete.

Proof. It is clear that $\Psi$ is not complete. Now, let $\left(\lambda_{n}\right)$ be a Cauchy sequence in $\Psi$ such that $\alpha\left(\lambda_{n}, \lambda_{n}, \lambda_{n+1}, \lambda_{n+1}\right) \geq 1$. Then $\lambda_{n}, \lambda_{n+1} \in\left\{1, \frac{1}{2}\right\}$. Since $\left(\lambda_{n}\right)$ is Cauchy in $\Psi$, we conclude that $\left(\lambda_{n}\right)$ has a constant tail 1 or has a constant tail $\frac{1}{2}$. In both cases, we conclude that $\left(\lambda_{n}\right)$ converges in $\Psi$ to 1 or $\frac{1}{2}$. Thus $\Psi$ is a coupled $-\alpha$-complete.

Example 10. Let $\Psi=[0,+\infty)$. Define a metric $\tau$ on $\Psi \times \Psi$ via $\tau(\lambda, \nu)=$ $|\lambda-\nu|$. Also, define the function $\alpha: \Psi^{4} \rightarrow[0,+\infty)$ via

$$
\alpha(\lambda, \nu, v, \zeta)= \begin{cases}\lambda, & \text { if } \lambda, \nu, v, \zeta>0 \\ 0, & \text { otherwise }\end{cases}
$$

and the mapping $F: \Psi \times \Psi \rightarrow \Psi$ via

$$
F(\lambda, \nu)= \begin{cases}\frac{|\lambda-\nu|}{2}, & \text { if } \lambda>0 \text { or } \nu>0 \\ 1, & \text { if } \lambda=\nu=0\end{cases}
$$

Then $F$ is a coupled- $\alpha$-continuous mapping which is not a continuous mapping.

Proof. Note that $\left(\frac{1}{n}, \frac{1}{n}\right) \rightarrow(0,0)$ but $\left(F\left(\frac{1}{n}, \frac{1}{n}\right)\right)$ doesn't converge to $F(0,0)$. So $F$ is not continuous. Now let $\left(\lambda_{n}, \nu_{n}\right)$ be a sequence in $\Psi \times \Psi$ that converges to an element $(\lambda, \nu) \in \Psi \times \Psi$ such that $\alpha\left(\lambda_{n}, \lambda_{n}, \lambda_{n+1}, \lambda_{n+1}\right) \geq 1$ and $\alpha\left(\nu_{n}, \nu_{n}, \nu_{n+1}, \nu_{n+1}\right) \geq 1$ for all $n \in \mathbf{N}$. So $\lambda_{n} \geq 1$ and $\nu_{n} \geq 1$ for all $n \in \mathbf{N}$ which implies that $\lambda \geq 1$ and $\nu \geq 1$. So the sequence $F\left(\lambda_{n}, \nu_{n}\right)=\frac{\left|\lambda_{n}-\nu_{n}\right|}{2}$ converges to $\frac{|\lambda-\nu|}{2}=F(\lambda, \nu)$ and hence $F$ is coupled $-\alpha$-continuous.

Definition 11. Let $F: \Psi \times \Psi \rightarrow \Psi$ be a mapping and $\alpha: \Psi^{4} \rightarrow \mathbf{R}$ be a function. The function $F$ is said to be coupled- $\alpha$-admissible if it satisfies the following conditions:

1. If $\kappa, \nu, v, \lambda \in \Psi$ such that $\alpha(\kappa, \nu, v, \lambda) \geq 1$, then

$$
\alpha(F(\kappa, \kappa), F(\nu, \nu), F(v, v), F(\lambda, \lambda)) \geq 1
$$

2. If $\lambda, \nu, v \in \Psi$ such that $\alpha(\lambda, \lambda, v, v) \geq 1$ and $\alpha(v, v, \nu, \nu) \geq 1$, then $\alpha(\lambda, \lambda, \nu, \nu) \geq 1$. 
Example 12. Let $\Psi=[0,+\infty)$. Define the function $\alpha: \Psi^{4} \rightarrow[0,+\infty)$ via

$$
\alpha(\lambda, \nu, v, \zeta)= \begin{cases}\lambda, & \text { if } \lambda, \nu, v, \zeta>0 \\ 0, & \text { otherwise }\end{cases}
$$

Also, define $F: \Psi \times \Psi \rightarrow \Psi$ via $F(\lambda, \nu)=\lambda+\nu$. Then $F$ is a coupled $-\alpha-$ admissible mapping.

Proof. Given $\lambda, \nu, v, \zeta \in \Psi$ be such that $\alpha(\lambda, \nu, v, \zeta) \geq 1$. So $\lambda, \nu, v, \lambda>0$. Note that

$$
\alpha(F(\lambda, \lambda), F(\nu, \nu), F(v, v), F(\zeta, \zeta))=\alpha(2 \lambda, 2 \nu, 2 v, 2 \zeta) \geq 1 .
$$

So Condition 1. of Definition 11 is satisfied. To verify Condition 2. of Definition 11 , let $\lambda, \nu, v \in \Psi$ be such that $\alpha(\lambda, \lambda, \nu, \nu) \geq 1$ and $\alpha(\nu, \nu, v, v) \geq 1$. Then we conclude that $\lambda, \nu, v>0$ and hence $\alpha(\lambda, \lambda, v, v)=\lambda \geq 1$. So $F$ is a coupled $-\alpha$-admissible mapping.

In order to make our work visible, we introduce the following definition:

Definition 13. Let $(\Psi, \tau)$ be a metric space. Let $F: \Psi \times \Psi \rightarrow \Psi$ be a mapping and $\alpha: \Psi^{4} \rightarrow[0,+\infty)$ be a function. Then the mapping $F$ is called coupled- $\alpha$-contraction of type (I) if there exist $a_{1}, a_{2}, a_{3}, a_{4}, a_{5}, a_{6} \in[0,1)$ with $\sum_{i=1}^{6} a_{i}<1$ such that if $\lambda, \nu, v, \lambda \in \Psi$ such that $\alpha(\lambda, \nu, v, \lambda) \geq 1$, then

$$
\begin{aligned}
\tau(F(\lambda, \nu), F(v, \lambda)) \leq & a_{1} \tau(\lambda, v)+a_{2} \tau(\nu, w)+a_{3} \tau(\lambda, F(\lambda, \nu)) \\
& +a_{4} \tau(\nu, F(\nu, \lambda))+a_{5} \tau(z, F(v, \lambda)) \\
& +a_{6} \tau(w, F(\lambda, v)) .
\end{aligned}
$$

Definition 14. Let $\Psi$ be a set. A point $(u, u) \in \Psi \times \Psi$ is called a FP of a mapping $F: \Psi \times \Psi \rightarrow \Psi$ if $F(u, u)=u$.

In the following results, we assume $(\Psi, \tau)$ to be a metric space and we consider a real-valued function $\alpha$ on $\Psi^{4}$ and a mapping $F: \Psi \times \Psi \rightarrow \Psi$.

Now, we ready to introduce and prove our main result:

Theorem 15. Suppose that the following conditions are satisfied:

1. $\tau$ is coupled- $\alpha$-complete;

2. $F$ is coupled- $\alpha$-continuous; 
3. $F$ is coupled- $\alpha$-contraction of type (I);

4. $F$ is coupled- $\alpha$-admissible;

5. There exists $\lambda_{0} \in \Psi$ such that

$$
\alpha\left(\lambda_{0}, \lambda_{0}, F\left(\lambda_{0}, \lambda_{0}\right), F\left(\lambda_{0}, \lambda_{0}\right)\right) \geq 1 \text {. }
$$

Then the mapping $F$ has a FP.

Proof. By (5), we choose $\lambda_{0} \in X$ be such that

$$
\alpha\left(\lambda_{0}, \lambda_{0}, F\left(\lambda_{0}, \lambda_{0}\right), F\left(\lambda_{0}, \lambda_{0}\right)\right) \geq 1
$$

Fixed $\lambda_{1}=F\left(\lambda_{0}, \lambda_{0}\right)$ and $\lambda_{2}=F\left(\lambda_{1}, \lambda_{1}\right)$, then $\alpha\left(\lambda_{0}, \lambda_{0}, \lambda_{1}, \lambda_{1}\right) \geq 1$. Condition (4) implies that

$$
\alpha\left(F\left(\lambda_{0}, \lambda_{0}\right), F\left(\lambda_{0}, \lambda_{0}\right), F\left(\lambda_{1}, \lambda_{1}\right), F\left(\lambda_{1}, \lambda_{1}\right)\right) \geq 1
$$

and hence

$$
\alpha\left(\lambda_{1}, \lambda_{1}, \lambda_{2}, \lambda_{2}\right) \geq 1
$$

By employing condition 3., we get

$$
\begin{array}{ll} 
& \tau\left(\lambda_{1}, \lambda_{2}\right)=\tau\left(F\left(\lambda_{0}, \lambda_{0}\right), F\left(\lambda_{1}, \lambda_{1}\right)\right) \\
\leq & a_{1} \tau\left(\lambda_{0}, \lambda_{1}\right)+a_{2} \tau\left(\lambda_{0}, \lambda_{1}\right)+a_{3} \tau\left(\lambda_{0}, F\left(\lambda_{0}, \lambda_{0}\right)\right) \\
& +a_{4} \tau\left(\lambda_{0}, F\left(\lambda_{0}, \lambda_{0}\right)\right)+a_{5} \tau\left(\lambda_{1}, F\left(\lambda_{1}, \lambda_{1}\right)\right)+a_{6} \tau\left(\lambda_{1}, F\left(\lambda_{1}, \lambda_{1}\right)\right) \\
= & a_{1} \tau\left(\lambda_{0}, \lambda_{1}\right)+a_{2} \tau\left(\lambda_{0}, \lambda_{1}\right)+a_{3} \tau\left(\lambda_{0}, \lambda_{1}\right) \\
& +a_{4} \tau\left(\lambda_{0}, \lambda_{1}\right)+a_{5} \tau\left(\lambda_{1}, \lambda_{2}\right)+a_{6} \tau\left(\lambda_{1}, \lambda_{2}\right) .
\end{array}
$$

From above inequalities, we reach to

$$
\tau\left(\lambda_{1}, \lambda_{2}\right) \leq \frac{a_{1}+a_{2}+a_{3}+a_{4}}{1-a_{5}-a_{6}} \tau\left(\lambda_{0}, \lambda_{1}\right)
$$

Let

$$
r=\frac{a_{1}+a_{2}+a_{3}+a_{4}}{1-a_{5}-a_{6}} .
$$

Then we have

$$
\tau\left(\lambda_{1}, \lambda_{2}\right) \leq r \tau\left(\lambda_{0}, \lambda_{1}\right)
$$


From Condition 4. and (2), we deduce that

$$
\alpha\left(F\left(\lambda_{1}, \lambda_{1}\right), F\left(\lambda_{1}, \lambda_{1}\right), F\left(\lambda_{2}, \lambda_{2}\right), F\left(\lambda_{2}, \lambda_{2}\right)\right) \geq 1
$$

and so, we have

$$
\alpha\left(\lambda_{2}, \lambda_{2}, \lambda_{3}, \lambda_{3}\right) \geq 1 .
$$

Similar arguments as above, we get

$$
\tau\left(\lambda_{2}, \lambda_{3}\right) \leq r \tau\left(\lambda_{1}, \lambda_{2}\right)
$$

Combining (4) and (6), we conclude that

$$
\tau\left(\lambda_{2}, \lambda_{3}\right) \leq r^{2} \tau\left(\lambda_{0}, \lambda_{1}\right) .
$$

By induction on $n$, we can show that $\alpha\left(\lambda_{n}, \lambda_{n}, \lambda_{n+1}, \lambda_{n+1}\right) \geq 1$ holds for all $n \in \mathbf{N} \cup\{\mathbf{0}\}$. Also, inductively we have

$$
\begin{aligned}
\tau\left(\lambda_{n}, \lambda_{n+1}\right) & \leq r \tau\left(\lambda_{n-1}, \lambda_{n}\right) \\
& \leq r^{2} \tau\left(\lambda_{n-2}, \lambda_{n-1}\right) \\
& \vdots \\
& \leq r^{n} \tau\left(\lambda_{0}, \lambda_{1}\right) .
\end{aligned}
$$

Now, we will show that $\left(\lambda_{n}\right)$ is Cauchy. Note that

$$
\alpha\left(\lambda_{n}, \lambda_{n}, \lambda_{n+1}, \lambda_{n+1}\right) \geq 1
$$

holds for all $n \in \mathbf{N} \cup\{\mathbf{0}\}$. So for $m>n$, we have

$$
\begin{aligned}
\tau\left(\lambda_{n}, \lambda_{m}\right) & \leq \tau\left(\lambda_{n}, \lambda_{n+1}\right)+\tau\left(\lambda_{n+1}, \lambda_{n+2}\right)+\ldots+\tau\left(\lambda_{m-1}, \lambda_{m}\right) \\
& \leq r^{n} \tau\left(\lambda_{0}, \lambda_{1}\right)+r^{n+1} \tau\left(\lambda_{0}, \lambda_{1}\right)+\ldots+r^{m-1} \tau\left(\lambda_{0}, \lambda_{1}\right) \\
& \leq \frac{r^{n}}{1-r} \tau\left(\lambda_{0}, \lambda_{1}\right) .
\end{aligned}
$$

Letting $n, m \rightarrow+\infty$ in above inequalities, we get that

$$
\lim _{n, m \rightarrow+\infty} \tau\left(\lambda_{n}, \lambda_{m}\right)=0 .
$$

So $\left(\lambda_{n}\right)$ is Cauchy. Since $(\Psi, \tau)$ is coupled $-\alpha-$ complete, then there exists $u \in \Psi$ such that $\lambda_{n} \rightarrow u$ as $n \rightarrow+\infty$. Since $F$ is coupled $-\alpha-$ continuous and $\alpha\left(\lambda_{n}, \lambda_{n}, \lambda_{n+1}, \lambda_{n+1}\right) \geq 1, \alpha\left(\lambda_{n+1}, \lambda_{n+1}, \lambda_{n+2}, \lambda_{n+2}\right) \geq 1$ for all $n \in \mathbf{N}$, we conclude that $\lambda_{n+1}=F\left(\lambda_{n}, \lambda_{n}\right) \rightarrow F(u, u)$. The uniqueness of limit implies that $u=F(u, u)$; that is, $(u, u)$ is a $\mathrm{FP}$ of $F$. 
From Theorem 15 we derived the following results:

Corollary 16. Suppose that the following conditions hold:

1. $\tau$ is coupled- $\alpha$-complete;

2. $F$ is coupled- $\alpha$-continuous;

3. $F$ is coupled- $\alpha$-admissible;

4. There exists $\lambda_{0} \in \Psi$ such that $\alpha\left(\lambda_{0}, \lambda_{0}, F\left(\lambda_{0}, \lambda_{0}\right), F\left(\lambda_{0}, \lambda_{0}\right)\right) \geq 1$;

5. There exist $a_{1}, a_{2} \in[0,1)$ with $a_{1}+a_{2}<1$ such that if $\lambda, \nu, v, \lambda \in \Psi$ such that $\alpha(\lambda, \nu, v, \lambda) \geq 1$, then

$$
\tau(F(\lambda, \nu), F(v, \lambda)) \leq a_{1} \tau(\lambda, v)+a_{2} \tau(\nu, w) .
$$

Then the mapping $F$ has a FP.

Corollary 17. Suppose that the following conditions hold:

1. $\tau$ is coupled- $\alpha$-complete;

2. $F$ is coupled- $\alpha$-continuous;

3. $F$ is coupled- $\alpha$-admissible;

4. There exists $\lambda_{0} \in \Psi$ such that $\alpha\left(\lambda_{0}, \lambda_{0}, F\left(\lambda_{0}, \lambda_{0}\right), F\left(\lambda_{0}, \lambda_{0}\right)\right) \geq 1$;

5. There exist $a_{1}, a_{2} \in[0,1)$ with $a_{1}+a_{2}<1$ such that if $\lambda, \nu, v, \lambda \in \Psi$ such that $\alpha(\lambda, \nu, v, \lambda) \geq 1$, then

$$
\tau(F(\lambda, \nu), F(v, \lambda)) \leq a_{1} \tau(\lambda, F(\lambda, \nu))+a_{2} \tau(\nu, F(\nu, \lambda)) .
$$

Then the mapping $F$ has a FP.

Corollary 18. Suppose that the following conditions hold:

1. $\tau$ is coupled- $\alpha$-complete;

2. $F$ is coupled- $\alpha$-continuous;

3. $F$ is coupled- $\alpha$-admissible; 
4. There exists $\lambda_{0} \in \Psi$ such that $\alpha\left(\lambda_{0}, \lambda_{0}, F\left(\lambda_{0}, \lambda_{0}\right), F\left(\lambda_{0}, \lambda_{0}\right)\right) \geq 1$;

5. There exist $a_{1}, a_{2} \in[0,1)$ with $a_{1}+a_{2}<1$ such that if $\lambda, \nu, v, \lambda \in \Psi$ such that $\alpha(\lambda, \nu, v, \lambda) \geq 1$, then

$$
\tau(F(\lambda, \nu), F(v, \lambda)) \leq a_{1} \tau(z, F(v, \lambda))+a_{2} \tau(w, F(\lambda, v)) .
$$

Then the mapping $F$ has a FP.

Now we introduce the notion of coupled- $\alpha$-contraction of type (II).

Definition 19. Let $(\Psi, \tau)$ be a metric space. Let $F: \Psi \times \Psi \rightarrow \Psi$ be a mapping and $\alpha: \Psi^{4} \rightarrow[0,+\infty)$ be a function. Then the mapping $F$ is called coupled- $\alpha$-contraction of type (II) if there exist $a_{1}, a_{2}, a_{3}, a_{4} \in[0,1)$ with $2 a_{1}+2 a_{2}<1$ such that if $\lambda, \nu, v, \lambda \in \Psi$ such that $\alpha(\lambda, \nu, v, \lambda) \geq 1$, then

$$
\begin{aligned}
\tau(F(\lambda, \nu), F(v, \lambda)) \leq & a_{1} \tau(\lambda, F(v, \lambda))+a_{2} \tau(\nu, F(\lambda, v)) \\
& +a_{3} \tau(z, F(\lambda, \nu))+a_{4} \tau(w, F(\nu, \lambda)) .
\end{aligned}
$$

Theorem 20. Suppose that the following conditions hold:

1. $\tau$ is coupled- $\alpha$-complete;

2. $F$ is coupled- $\alpha$-continuous;

3. $F$ is coupled- $\alpha$-contraction of type (II);

4. $F$ is coupled- $\alpha$-admissible;

5. There exists $\lambda_{0} \in \Psi$ such that $\alpha\left(\lambda_{0}, \lambda_{0}, F\left(\lambda_{0}, \lambda_{0}\right), F\left(\lambda_{0}, \lambda_{0}\right)\right) \geq 1$.

Then the mapping $F$ has a FP.

Proof. By fixing $\lambda_{n+1}=F\left(\lambda_{n}, \lambda_{n}\right)$ and starting with condition (5), we can construct a sequence $\left(\lambda_{n}\right)$ of $\Psi$ such that

$$
\alpha\left(\lambda_{n}, \lambda_{n}, \lambda_{n+1}, \lambda_{n+1}\right) \geq 1
$$

By employing condition (3), we have

$$
\begin{aligned}
\tau\left(\lambda_{n+1}, \lambda_{n+2}\right) & =\tau\left(F\left(\lambda_{n}, \lambda_{n}\right), F\left(\lambda_{n+1}, \lambda_{n+1}\right)\right) \\
\leq & a_{1} \tau\left(\lambda_{n}, F\left(\lambda_{n+1}, \lambda_{n+1}\right)\right)+a_{2} \tau\left(\lambda_{n}, F\left(\lambda_{n+1}, \lambda_{n+1}\right)\right)
\end{aligned}
$$




$$
\begin{aligned}
& +a_{3} \tau\left(\lambda_{n+1}, F\left(\lambda_{n}, \lambda_{n}\right)\right)+a_{4} \tau\left(\lambda_{n+1}, F\left(\lambda_{n}, \lambda_{n}\right)\right) \\
= & a_{1} \tau\left(\lambda_{n}, \lambda_{n+2}\right)+a_{2} \tau\left(\lambda_{n}, \lambda_{n+2}\right) .
\end{aligned}
$$

Applying triangle inequality to the above inequality, we get

$$
\tau\left(\lambda_{n+1}, \lambda_{n+2}\right) \leq \frac{a_{1}+a_{2}}{1-a_{1}-a_{2}} \tau\left(\lambda_{n}, \lambda_{n+1}\right) .
$$

To facilitate our computation, we let $r=\frac{a_{1}+a_{2}}{1-a_{1}-a_{2}}$. Thus we have

$$
\tau\left(\lambda_{n+1}, \lambda_{n+2}\right) \leq r \tau\left(\lambda_{n}, \lambda_{n+1}\right) .
$$

Repeating (9) n-times, we deduce that

$$
\tau\left(\lambda_{n}, \lambda_{n+1}\right) \leq r^{n} \tau\left(\lambda_{0}, \lambda_{1}\right)
$$

For $m>n$ and applying triangle inequality, we get

$$
\tau\left(\lambda_{n}, \lambda_{m}\right) \leq \frac{r^{n}}{1-r} \tau\left(\lambda_{0}, \lambda_{n}\right)
$$

By letting $n, m \rightarrow+\infty$ in above inequality, we conclude that $\left(\lambda_{n}\right)$ is Cauchy. Therefore, $\lambda_{n} \rightarrow u \in \Psi$. Since $\alpha\left(\lambda_{n}, \lambda_{n}, \lambda_{n+1}, \lambda_{n+1}\right) \geq 1$ and $\Psi$ is coupled $-\alpha-$ complete, we have $\left(\lambda_{n+1}=F\left(\lambda_{n}, \lambda_{n}\right)\right)$ converges to $u$ and $F(u, u)$. Uniqueness of limit implies that $F(u, u)=u$; that is, $u$ is a coupled $\mathrm{FP}$ of $F$.

Now we utilize Theorem 20 to derive the following results:

Corollary 21. Suppose that the following conditions hold:

1. $\tau$ is coupled- $\alpha$-complete;

2. $F$ is coupled- $\alpha$-continuous;

3. $F$ is coupled- $\alpha$-admissible;

4. There exists $\lambda_{0} \in \Psi$ such that $\alpha\left(\lambda_{0}, \lambda_{0}, F\left(\lambda_{0}, \lambda_{0}\right), F\left(\lambda_{0}, \lambda_{0}\right)\right) \geq 1$;

5. There exist $a_{1}, a_{2} \in[0,1)$ with $2 a_{1}+2 a_{2}<1$ such that if $\lambda, \nu, v, \lambda \in \Psi$ such that $\alpha(\lambda, \nu, v, \lambda) \geq 1$, then

$$
\tau(F(\lambda, \nu), F(v, \lambda)) \leq a_{1} \tau(\lambda, F(v, \lambda))+a_{2} \tau(\nu, F(\lambda, v)) .
$$

Then the mapping $F$ has a FP. 
Corollary 22. Suppose that the following conditions hold:

1. $\tau$ is coupled- $\alpha$-complete;

2. $F$ is coupled- $\alpha$-continuous;

3. $F$ is coupled- $\alpha$-admissible;

4. There exists $\lambda_{0} \in \Psi$ such that $\alpha\left(\lambda_{0}, \lambda_{0}, F\left(\lambda_{0}, \lambda_{0}\right), F\left(\lambda_{0}, \lambda_{0}\right)\right) \geq 1$;

5. There exist $a_{1}, a_{2} \in[0,1)$ such that if $\lambda, \nu, v, \lambda \in \Psi$ such that $\alpha(\lambda, \nu, v, \lambda) \geq$ 1 , then

$$
\tau(F(\lambda, \nu), F(v, \lambda)) \leq a_{1} \tau(z, F(\lambda, \nu))+a_{2} \tau(w, F(\nu, \lambda)) .
$$

Then the mapping $F$ has a FP.

Corollary 23. Suppose that the following conditions hold:

1. $\tau$ is coupled- $\alpha$-complete;

2. $F$ is coupled- $\alpha$-continuous;

3. $F$ is coupled- $\alpha$-admissible;

4. There exists $\lambda_{0} \in \Psi$ such that $\alpha\left(\lambda_{0}, \lambda_{0}, F\left(\lambda_{0}, \lambda_{0}\right), F\left(\lambda_{0}, \lambda_{0}\right)\right) \geq 1$;

5. There exist $a_{1}, a_{2} \in[0,1)$ with $2 a_{1}<1$ such that if $\lambda, \nu, v, \lambda \in \Psi$ such that $\alpha(\lambda, \nu, v, \lambda) \geq 1$, then

$$
\tau(F(\lambda, \nu), F(v, \lambda)) \leq a_{1} \tau(\lambda, F(v, \lambda))+a_{2} \tau(z, F(\lambda, \nu)) .
$$

Then the mapping $F$ has a FP.

Corollary 24. Suppose that the following conditions hold:

1. $\tau$ is coupled- $\alpha$-complete;

2. $F$ is coupled- $\alpha$-continuous;

3. $F$ is coupled- $\alpha$-admissible;

4. There exists $\lambda_{0} \in \Psi$ such that $\alpha\left(\lambda_{0}, \lambda_{0}, F\left(\lambda_{0}, \lambda_{0}\right), F\left(\lambda_{0}, \lambda_{0}\right)\right) \geq 1$; 
5. There exist $a_{1}, a_{2} \in[0,1)$ with $2 a_{1}<1$ such that if $\lambda, \nu, v, \lambda \in \Psi$ such that $\alpha(\lambda, \nu, v, \lambda) \geq 1$, then

$$
\tau(F(\lambda, \nu), F(v, \lambda)) \leq a_{1} \tau(\nu, F(\lambda, v))+a_{2} \tau(\lambda, F(\nu, \lambda)) .
$$

Then the mapping $F$ has a FP.

\section{References}

[1] T. Abdeljawad, Meir-Keeler $\alpha$-contractive fixed and common fixed point theorems. Fixed Point Theory Appl., 2013 (2013), 10 pages.

[2] T. Abdeljawad, J. Alzabut, A. Mukheimer, Y. Zaidan, Best proximity points for cyclical contraction mappings with 0-boundedly compact decompositions, J. of Computational Analysis and Applications, 15, No 4 (2013), 678-685.

[3] T. Abdeljawad, J. Alzabut, A. Mukheimer, Y. Zaidan, Banach contraction principle for cyclical mappings on partial metric spaces, Fixed Point Theory and Applications, 2012 (2012), Art. ID 154.

[4] K. Abodayeh, W Shatanawi, A Bataihah, AH Ansari, Some fixed point and common fixed point results through $\Omega$-distance under nonlinear contractions, Gazi University J. of Science, 30, No 1 (2017), 293-302.

[5] A. Al-Rawashdeha, H. Aydi, F. Abdelbasset, S. Sahmim, W. Shatanawi, On common fixed points for $\alpha-F$-contractions and applications, J. Nonlinear Sci. Appl., 9 (2016), 3445-3458.

[6] A.H. Ansari, J. Kaewcharoen, C-class functions and fixed point theorems for generalized $\alpha-\eta-\psi-\varphi-F$-contraction type mapping in $\alpha-\eta$-complete metric space, Nonlinear Sci. Appl., 9 (2016), 4177-4190.

[7] H. Aydi, E. Karapinar, and W. Shatanawi, Tripled common fixed point results for generalized contractions in ordered generalized metric spaces, Fixed Point Theory and Applications, 2012, No 1 (2012), Art. ID 101.

[8] H. Aydi, W. Shatanawi, M. Postolache, Z. Mustafa, and N. Tahat, Theorems for Boyd-Wong type contractions in ordered metric spaces, Abstract and Applied Analysis 2012 (2012), Art. ID 359054, 14 pages.

[9] S. Banach, Sur les opérations dans les ensembles et leur application aux equations itegrales, Fundam. Math. 3 (1922), 133-181. 
[10] Y.J. Cho, Z. Kadelburg, R. Saadati, and W. Shatanawi, Coupled fixed point theorems under weak contractions, Discrete Dynamics in Nature and Society, 2012 (2012), Art. ID 184534, 9 pages; doi:10.1155/2012/184534.

[11] N. Hussain, M. Arshad, A. Shoaib, Fahimuddin, Common fixed point results for $\alpha-\psi$-contractions on a metric space endowed with graph, J. of Inequalities Appl., 2014 (2014), Art. ID 136.

[12] N. Hussain, M.A. Kutbi, P. Salimi, Fixed point theory in $\alpha$-complete metric spaces with applications, Abstr. Appl. Anal., 2014 (2014), 11 pages, $1,1.16,1.18$.

[13] N. Hussain, P. Salimi and A. Latif, Fixed point results for single and setvalued a $\alpha-\eta-\psi-$ contractive mappings, Fixed Point Theory Appl., 2013 (2013), Art. ID 212.

[14] E. Karapinar, P. Kumam and P. Salimi, On $\alpha-\psi-$ Meir-Keeler contractive mappings, Fixed Point Theory Appl., 2013 (2013), Art. ID 94.

[15] H. Qawagneh, M.S. Noorani, W. Shatanawi, K. Abodayeh and H. Alsamir, Fixed point for mappings under contractive condition based on simulation functions and cyclic $(\alpha-\beta)$-admissibility, J. of Mathematical Analysis, 9, No 1 (2018), 38-51.

[16] T. Qawasmeh, W. Shatanawi, A. Bataihah and A. Tallafha, Common fixed point results for rational $(\alpha, \beta)_{\varphi}$-contractions in complete quasi metric spaces, Mathematics, 7, No 5 (2019), Art. ID 392; doi:10.3390/math7050392.

[17] P. Salimi, A. Latif and N. Hussain, Modified $\alpha-\psi$-Contractive mappings with applications, Fixed Point Theory Appl., 2013 (2013), Art. ID 151.

[18] B. Samet, C. Vetro, P. Vetro, Fixed point theorems for an $\alpha-\psi$-contractive type mappings, Nonlinear Anal., 75 (2012), 2154-2165.

[19] W. Shatanawi, Fixed and common fixed point theorems in frame of quasi metric spaces under contraction condition based on ultra distance functions, Nonlinear Analysis: Modelling and Control, 23, No 5 (2018), page 724.

[20] W. Shatanawi, K. Abodayeh, Common fixed point for mappings under contractive condition based on almost perfect functions and $\alpha$-admissibility, Nonlinear Functional Analysis and Applications, 23 (2018), 247-257.

[21] W. Shatanawi, and K. Abodayeh, Fixed point results for mapping of nonlinear contractive conditions of $\alpha$-admissibility form, IEEE Access, 7 (2019) 50280-50286; doi:10.1109/ACCESS.2019.2910794. 
[22] W. Shatanawi, and K. Abodayeh, Common fixed point under nonlinear contractions on quasi metric spaces, Mathematics, 2019, No 7 (2019), Art. ID 453.

[23] W. Shatanawi, and K. Abodayeh, Some fixed and common fixed point results in g-metric spaces which cannot be obtained from metric spaces, Boletim da Sociedade Paranaense de Matematica, 38, No 6 (2020), 4-51.

[24] W. Shatanawi, K. Abodayeh, and A. Bataihah, Fixed point theorem through $\Omega$-distance of Suzuki type contraction condition, Gazi University J. of Science, 29, No 1 (2016), 129-133.

[25] W. Shatanawi, K. Abodayeh, A. Mukheimer, Some fixed point theorems in extended b-metric spaces, U.P.B. Sci. Bull., Series A, 80, No 4 (2018).

[26] W. Shatanawi, A. Al-Rawashdeh, Common fixed points of almost generalized $(\psi, \phi)$-contractive mappings in ordered metric spaces, Fixed Point Theory and Applications, 2012, No 1 (2012), Art. ID 80.

[27] W. Shatanawi, Z Mustafa, N Tahat, Some coincidence point theorems for nonlinear contraction in ordered metric spaces, Fixed point Theory and applications, 2011, No 1 (2011), Art. ID 68.

[28] W. Shatanawi, Z.D. Mitrovic, N. Hussain and S. Radenovic, On generalized hardy rogers type $\alpha$-admissible mappings in cone b-metric spaces over Banach algebras, Symmetry, 2020, No 12 (2020), Art. ID 81; doi:10.3390/sym12010081.

[29] W. Shatanawi, M.S. MD Noorani, H. Alsamir, A. Bataihah, Fixed and common fixed point theorems in partially ordered quasimetric spaces, $J$. Math. Computer Sci., 16 (2016), 516-528.

[30] W. Shatanawi, M. Postolache, Coincidence and fixed point results for generalized weak contractions in the sense of Berinde on partial metric spaces, Fixed Point Theory and Applications 2013 (2013), Art. ID 54.

[31] W. Shatanawi, M. Postolache, Some fixed-point results for a-weak contraction in-metric spaces, Abstract and Applied Analysis, 2012 (2012), Art. ID 815870, 19 pages. 